\title{
ON THE ROBUSTNESS OF STRUCTURAL RISK OPTIMIZATION WITH RESPECT TO EPISTEMIC UNCERTAINTIES
}

\author{
Andre T. Beck, W. J. S. Gomes, \& F. A. V. Bazan \\ Structural Engineering Department, EESC, University of São Paulo, Av. Trabalhador São- \\ carlense, 400, 13566-590 São Carlos, SP, Brazil \\ Original Manuscript Submitted: 4/27/2011; Final Draft Received: 7/26/2011
}

\begin{abstract}
In the context of structural design, risk optimization allows one to find a proper point of balance between the concurrent goals of economy and safety. Risk optimization involves the minimization of total expected costs, which include expected costs of failure. Expected costs of failure are evaluated from nominal failure probabilities, which reflect the analyst's degree of belief in the structure's performance. Such failure probabilities are said to be nominal because they are evaluated from imperfect and/or incomplete mechanical, mathematical and probabilistic models. Hence, model uncertainty and other types of epistemic uncertainties are likely to compromise the results of risk optimization. In this paper, the concept of robustness is employed in order to find risk optimization solutions which are less sensitive to epistemic uncertainties. The investigation is based on a simple but illustrative problem, which is built from an elementary but fundamental structural (load-resistance) reliability problem. Intrinsic or aleatoric uncertainties, which can be quantified probabilistically and modeled as random variables or stochastic processes, are incorporated in the underlying structural reliability problem. Epistemic uncertainties that can only be quantified possibilistically are modeled as fuzzy variables, based on subjective judgment. These include uncertainties in random load and resistance variables, in the nominal (calculated) failure probabilities and in the nominal costs of failure. The risk optimization problem is made robust with respect to the whole fuzzy portfolio of epistemic uncertainties. An application example, involving optimization of partial safety factors for the codified design of steel beams under bending, is also presented. In general, results obtained herein show that the robust formulation leads to optimal structural configurations which are more conservative, present higher nominal costs but which are less sensitive to epistemic uncertainties, in comparison to the non-robust optimum structures. This is especially true for larger levels of intrinsic uncertainties (in the underlying reliability problem) and for greater costs of failure. The essential result of robust optimization is also shown to be insensitive to reasonable variations of expert confidence: the robust solution is more conservative and more expensive, but also less sensitive to epistemic uncertainties. The more pessimistic the expert, the more conservative is the robust solution he gets, in comparison to the nominal, non-robust solution.
\end{abstract}

KEY WORDS: risk analysis, representation of uncertainty, robust optimization, structural reliability, fuzzy variables, epistemic uncertainty

\section{INTRODUCTION}

Results of structural optimization should be robust with respect to the uncertainties inherently present in material properties and in structural loads. This notion has led to the development of different approaches to structural optimization: stochastic or robust optimization [1-4], fuzzy optimization [5], and reliability-based structural optimization [6-15]. Generally, robust formulations yield multi-objective optimization problems, where mean system performance should be maximized and performance variance should be minimized. The balance between these objectives is a subjective

\footnotetext{
${ }^{*}$ Correspond to Andre T. Beck, E-mail: atbeck@ sc.usp.br, URL: http://www.set.eesc.usp.br/public/pessoas/professor.php?id=3
} 
Beck, Gomes, \& Bazan

choice of the analyst. In reliability-based design optimization, a probabilistic measure of system performance, also subjectively chosen by the analyst, is used as the design constraint. Consequences of failure are not necessarily taken into account in these formulations. However, the main effect of uncertainties is the possibility of reaching a state of undesirable system performance. This possibility can be measured in terms of probability, and then multiplied by the cost (monetary measure of the consequences) of failure. The resulting term, also known as the expected cost of failure, can be incorporated in the objective function, leading to an unconstrained optimization problem: minimization of total expected costs [16-21]. This formulation, also known as risk optimization [22], allows one to find the optimum point of compromise between different possible failure modes, as well as the optimum safety margin with respect to each failure mode [23].

However, expected costs of failure are evaluated from nominal failure probabilities, which reflect the analyst's degree of belief in the structure's performance. Such failure probabilities are said to be nominal because they are evaluated from imperfect and/or incomplete mechanical, mathematical, and probabilistic models. Hence, model uncertainty and other types of epistemic uncertainties are likely to affect the solution of risk optimization problems.

In this paper, the concept of robustness is employed in order to find risk optimization solutions that are less sensitive to epistemic uncertainties. Intrinsic or aleatoric uncertainties, which can be quantified probabilistically and modeled as random variables or stochastic processes, are incorporated in the underlying structural reliability problem. Epistemic uncertainties that can only be quantified possibilistically are modeled as fuzzy variables, based on subjective judgment. The risk optimization problem is made robust with respect to the whole fuzzy portfolio of epistemic uncertainties.

The article is laid out as follows. A general formulation of risk optimization is presented in Section 2. A simple but illustrative problem is also presented in this section. The types of uncertainties affecting risk optimization problems are described in Section 3. A fuzzy representation of epistemic uncertainties is presented in Section 4. A robust risk optimization problem is formulated in Section 5, and applied in the solution of a practical engineering problem in Section 6. The effects of expert confidence are investigated in Section 7. Concluding remarks are presented in Section 8 .

\section{FORMULATION OF RISK OPTIMIZATION PROBLEM}

\subsection{General Formulation}

Let $\mathbf{X}$ and $\mathbf{d}$ be vectors of structural system parameters. Vector $\mathbf{X}$ represents all random system parameters, and includes geometric characteristics, resistance properties of materials or structural members, and loads. Some of these parameters are random in nature; others cannot be defined deterministically due to uncertainty. Typically, resistance parameters can be represented as random variables and loads are modeled as stochastic processes of time. Vector $\mathbf{d}$ contains system design variables (assumed deterministic), like partial safety factors, design life, parameters of the inspection and maintenance programs, etc. Vector $\mathbf{d}$ may include parameters of the random variables in $\mathbf{X}$ (e.g., the mean of a random variable).

The existence of uncertainty implies risk, that is, the possibility of undesirable structural responses. The boundary between desirable and undesirable structural responses is given by limit state equations $g(\mathbf{d}, \mathbf{x})=0$, such that:

$$
\begin{aligned}
& D_{f}=\{\mathbf{d}, \mathbf{x} \mid g(\mathbf{d}, \mathbf{x}) \leq 0\} \text { is the failure domain, } \\
& D_{s}=\{\mathbf{d}, \mathbf{x} \mid g(\mathbf{d}, \mathbf{x})>0\} \text { is the safety domain. }
\end{aligned}
$$

Each limit state describes one possible failure mode of the structure, either in terms of performance (serviceability) or ultimate capacity. The probability of undesirable structural response, or probability of failure, is given by

$$
P_{f}(\mathbf{d})=P[g(\mathbf{d}, \mathbf{X}) \leq 0],
$$

where $P[$.$] stands for probability. In this article, simple problems are considered and probabilities of failure are evalu-$ ated using traditional structural reliability methods such as the first-order reliability method (FORM) and second-order reliability method (SORM) [24, 25]. For more elaborate problems, advanced simulation techniques are available [15]. 
The life-cycle cost of a structural system subject to risk can be decomposed in an initial or construction cost, cost of operation, cost of inspections and maintenance, cost of disposal, and expected cost of failure. For a given failure mode, the expected cost of failure $\left(\mathrm{J}^{\text {expected }}\right)$ —or failure risk — is given by the product of failure cost $\left(\mathrm{J}^{\text {failure }}\right)$ by failure probability:

$$
\mathbf{J}^{\text {expected }}(\mathbf{d})=\mathbf{J}^{\text {failure }}(\mathbf{d}) P_{f}(\mathbf{d}) \text {. }
$$

Failure costs include the costs of repairing or replacing damaged structural members, removing a collapsed structure, rebuilding it, cost of unavailability, cost of compensation for injury or death of employees or general users, penalties for environmental damage, etc. All failure consequences have to be expressed in terms of monetary units, which can be a problem when dealing with human injury, human death, or environmental damage. Evaluation of such failure consequences in terms of the amount of compensation payoffs allows the problem to be formulated, without directly addressing matters about the value of human life [26, 27].

For each structural component and for each failure mode, there is a corresponding failure cost term. The total (life-cycle) expected cost of a structural system becomes

$$
\begin{aligned}
\mathrm{J}^{\text {total }}(\mathbf{d}) & =\mathrm{J}^{\text {initial or construction }}(\mathbf{d}) \\
& +\mathrm{J}^{\text {operation }}(\mathbf{d}) \\
& +\mathrm{J}^{\text {inspection and maintenance }}(\mathbf{d}) \\
& +\mathrm{J}^{\text {disposal }}(\mathbf{d}) \\
& +\sum_{\text {failure modes }} \mathrm{J}^{\text {failure }}(\mathbf{d}) P_{f}(\mathbf{d}) .
\end{aligned}
$$

The initial or construction cost increases with the safety coefficients used in the design and with the practiced level of quality assurance. More safety in operation involves more safety equipment, more redundancy, and more conservatism in structural operation. Inspection cost depends on intervals, quality of equipment, and choice of inspection method. Maintenance costs depend on maintenance plan, frequency of preventive maintenance, etc. When the overall level of safety is increased, most cost terms increase, but the expected costs of failure are reduced.

Any change in $\mathbf{d}$ that affects cost terms is likely to affect the expected cost of failure. Changes in $\mathbf{d}$ that reduce costs may result in increased failure probabilities; hence, increased expected costs of failure. Reduction in expected costs of failure can be achieved by targeted changes in $\mathbf{d}$, which generally increase costs. This compromise between safety and costs is typical of engineering systems.

The structural risk optimization problem can be stated as finding

$$
\mathbf{d}^{*}=\arg \min \left\{\mathbf{J}^{\text {total }}(\mathbf{d}): \mathbf{d} \in W\right\},
$$

where $W=\left\{\mathbf{d}_{\text {inf }} \leq \mathbf{d} \leq \mathbf{d}_{\text {sup }}\right\}$; and where $\mathbf{d}_{\text {inf }}$ and $\mathbf{d}_{\text {sup }}$ are the lower and upper bounds of the design variables.

\subsection{Elementary Structural Risk Optimization Problem}

The risk optimization formulation just presented is too general for a practical study of the effects of epistemic uncertainties. In the present paper, an elementary form of this problem is studied. This elementary form involves a timeinvariant reliability problem (only random variables); hence, no operation, inspection, or maintenance costs. Moreover, the elementary risk optimization problem is based on the fundamental (capacity-demand or stress-strength) reliability problem, which involves only two random variables: $R$ for resistance or capacity and $S$ for stress or demand. For this elementary but fundamental problem, the limit state equation is

$$
g(R, S)=R-S=0 .
$$

An analytical solution to this elementary problem is available when both random variables have normal (Gaussian) distributions [24]. Hence, it is assumed herein that:

$$
\begin{aligned}
& R \sim N\left(\mu_{R}, \sigma_{R}\right) \\
& S \sim N\left(\mu_{S}, \sigma_{S}\right),
\end{aligned}
$$

Volume 2, Number 1, 2012 
where $\mu$ and $\sigma$ are the mean and standard deviation of the respective variables. Hence, the failure probability can be calculated in closed form as

$$
P_{f}=\Phi(-\beta)=\Phi\left(-\frac{\mu_{R}-\mu_{S}}{\sqrt{\sigma_{R}^{2}+\sigma_{S}^{2}}}\right) .
$$

where $\beta$ is the reliability index and $\Phi($.$) is the cumulative distribution function for a standard normal variable Y \sim$ $N(0,1)$. In order to formulate the design problem, the central safety factor $\lambda$ is introduced:

$$
\lambda=\frac{\mu_{R}}{\mu_{S}} ; \text { hence, } \mu_{R}=\lambda \mu_{S}, \lambda>1 \text {. }
$$

To simplify the problem even further, it will be assumed that the coefficient of variation (c.o.v.) ( $\rho$ ) for both variables $R$ and $S$ is the same:

$$
\rho_{S}=\frac{\sigma_{S}}{\mu_{S}}=\rho_{R}=\rho ; \text { hence, } \sigma_{R}=\rho \lambda \mu_{S} .
$$

However, it should be acknowledged that in most real civil engineering applications the c.o.v. for loads is generally larger than the c.o.v. of resistance variables. Introducing Eqs. (10) and (11) in to Eq. (9), one obtains, after some elementary algebra,

$$
P_{f}(\lambda, \rho)=\Phi\left(\frac{1-\lambda}{\rho \sqrt{1+\lambda^{2}}}\right) .
$$

In Eq. (12), the central safety factor $\lambda$ is the (risk) optimization variable and $\rho$ is a measure of the problem's uncertainty. For this elementary risk optimization problem there is only one possible failure mode. The initial or construction cost is assumed proportional to $\lambda$, and the cost of failure is $k \lambda$, where $k$ is an additional problem variable. Hence, the objective function becomes

$$
\mathbf{J}^{\text {total }}(\lambda, k, \rho)=\mathbf{J}(\lambda, k, \rho)=\mathbf{J}^{\text {initial }}(\lambda)+\mathbf{J}^{\text {expected }}(\lambda, k, \rho)=\lambda+k \lambda P_{f}(\lambda, \rho) .
$$

The elementary but fundamental risk optimization problem is to find

$$
\lambda^{*}=\underset{\lambda}{\arg \min }\left\{\mathbf{J}^{\text {total }}(\lambda, k, \rho): \lambda>1\right\} .
$$

The Karush-Kuhn-Tucker necessary condition $[28,29]$ for $\lambda^{*}$ to be a solution to the above problem is

$$
\frac{\partial \mathrm{J}(\lambda, k, \rho)}{\partial \lambda}=1+k \lambda \frac{\partial P_{f}(\lambda, \rho)}{\partial \lambda}=1+k\left[\Phi\left(\frac{1-\lambda}{\rho \sqrt{1+\lambda^{2}}}\right)-\frac{\lambda}{\rho \sqrt{1+\lambda^{2}}} \phi\left(\frac{1-\lambda}{\rho \sqrt{1+\lambda^{2}}}\right)\left(1-\frac{\lambda(\lambda-1)}{\left(1+\lambda^{2}\right)}\right)\right]=0 .
$$

\subsection{Variants of the Elementary Risk Optimization Problem}

The elementary but fundamental risk optimization problem stated in Eqs. (12)-(14) has many variants, according to the values of the c.o.v. $(\rho)$ and the failure cost multiplier $(k)$. Typical values of $\rho$ for civil engineering problems are within $\{0.05 \leq \rho \leq 0.5\}$. The cost of failure for civil engineering structures, following the Joint Committee on Structural Safety [30], is classified as:

$$
\begin{array}{cl}
\text { Minor consequences: } & k<2, \\
\text { Moderare consequences: } & 2 \leq k<5, \\
\text { Severe consequences: } & 5 \leq k<10, \\
\text { Extreme consequences: } & 10 \leq k .
\end{array}
$$

In the design of structures with extreme failure consequences $(k \geq 10)$, a detailed risk-benefit analysis is suggested [30]. Since this is the objective of risk optimization, this range of values $\{10 \leq k \leq 20\}$ is investigated herein.

Figure 1 illustrates the objective (cost) function [Eq. (13)] for different, typical values of $\rho$ and $k$. Figure 2 shows the derivative with respect to $\lambda$ of these functions, where the roots $\left(\lambda^{*}\right)$ of Eq. (15) can be observed. In Fig. 1 it 

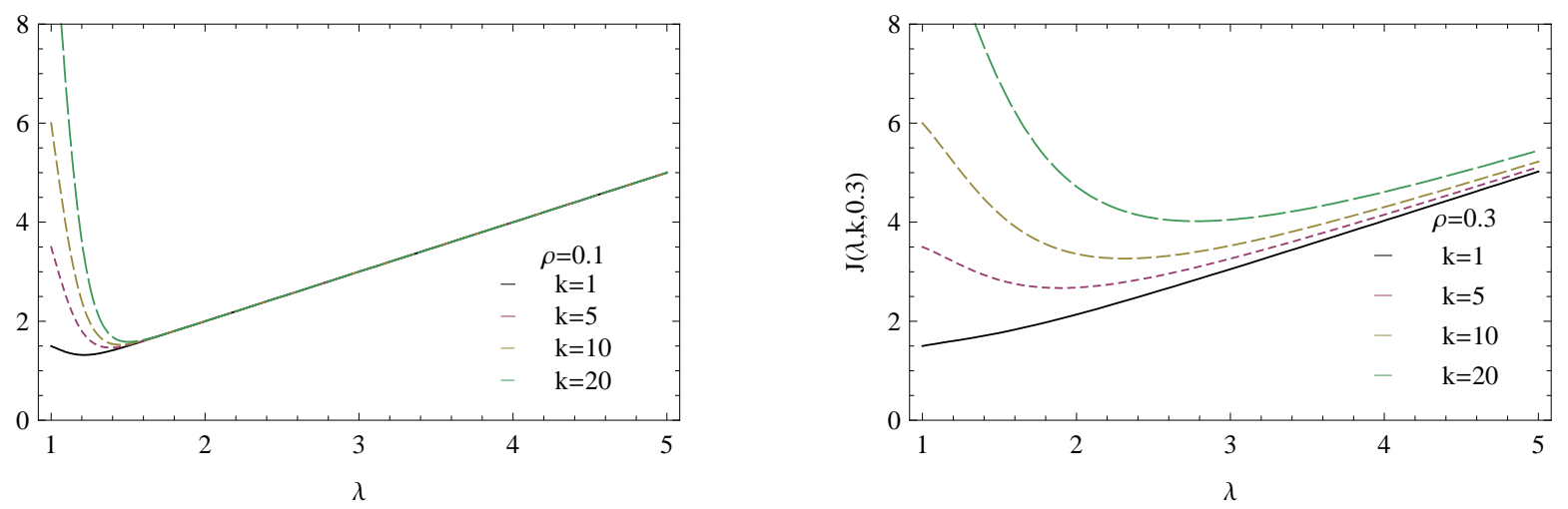

FIG. 1: Cost functions $\mathrm{J}()$ in terms of c.o.v. ( $\rho)$, failure cost $(k)$, and optimization parameter $(\lambda)$.
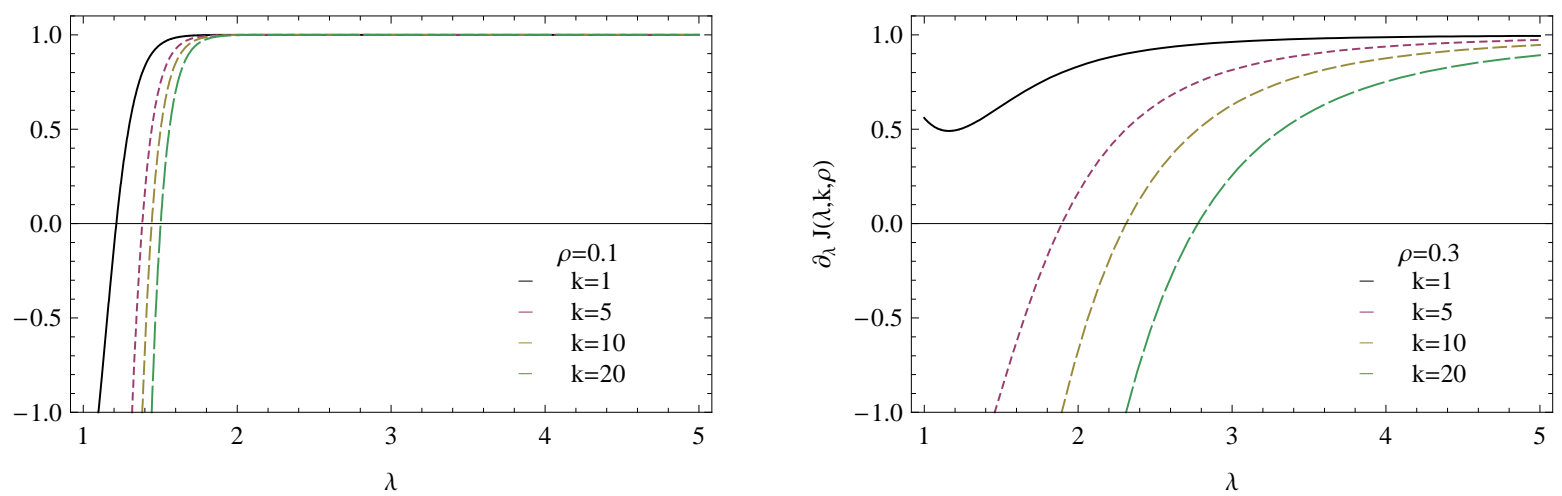

FIG. 2: Derivative of cost functions $J()$ in terms of optimization parameter $(\lambda)$.

can be observed that for $\rho=0.1$ the cost functions are sharper, having more clearly defined minima. In Fig. 2 it is also observed that the minima do not change much with respect to the failure cost multiplier $k$. On the other hand, for $\rho=0.3$ cost functions are broader, and their minima are not clearly observable. The minimum points change significantly with failure cost $k$, but optimum costs do not change much within different $\lambda^{*}$. Hence, it is observed that results of risk optimization $\left[\lambda^{*}\right.$ and $\left.\mathbf{J}\left(\lambda^{*}\right)\right]$ are less sensitive to (external) perturbations when problem uncertainty is larger (larger $\rho$ ) and when failure consequences are smaller.

\section{UNCERTAINTIES AFFECTING RISK OPTIMIZATION PROBLEM}

Different types of uncertainties can affect the performance of engineering systems. One important distinction is between intrinsic and epistemic uncertainties. Intrinsic or aleatoric uncertainty is due to the inherent variability of physical processes, and includes uncertainty in resistance and other parameters of structural materials, load actions (environmental actions, in particular), structural dimensions, etc. Epistemic uncertainties are related to the level of knowledge about the problem, and include statistical uncertainties, model errors, and phenomenological uncertainty. Intrinsic uncertainty is unavoidable and largely irreducible, whereas epistemic uncertainty can, in principle, be reduced by improving the level of knowledge about the problem. Broadly speaking, intrinsic uncertainties can be quantified and represented as random variables or stochastic processes, as do some types of epistemic uncertainties. This is called the probabilistic quantification of uncertainties. However, due to lack of knowledge, most forms of epistemic uncertainty cannot be quantified or represented probabilistically. Epistemic uncertainties admit a possibilistic representation using, for example, interval analysis or fuzzy number approaches. The interested reader is referred to [31] for a general discussion on the aleatoric/epistemic classification of uncertainties. 
In general robust optimization applications, no distinction needs to be made between intrinsic and epistemic uncertainties [1]. In the present application to risk optimization, it is assumed that all uncertainties (either intrinsic or epistemic) that can be quantified probabilistically are included in the formulation of the underlying reliability problem [Eq. (3)]. Hence, robustness of the risk optimization problem is sought with respect to epistemic uncertainties that cannot be described probabilistically. In the present paper, these uncertainties are described using fuzzy variables $[5,32]$.

In order to model the effects of epistemic uncertainties, an augmented version of the fundamental risk optimization problem is introduced:

$$
\begin{gathered}
\mathbf{J}_{p}=\mathbf{J}_{p}^{\text {total }}(\lambda, k, \rho, \delta m, \delta k, \delta \rho)=\lambda+\lambda k(1+\delta k) 10^{\delta m} P_{f}[\lambda, \rho(1+\delta \rho)], \\
\lambda_{p}^{*}=\underset{\lambda}{\arg \min }\left\{\mathbf{J}_{p}^{\text {total }}(\lambda, k, \rho, \delta m, \delta k, \delta \rho): \lambda>1\right\} .
\end{gathered}
$$

In Eq. (17), $\delta \rho$ is a perturbation in the c.o.v. (reflecting problem uncertainty) and $\delta k$ is a perturbation on the failure cost term. These quantities are discussed in the sequence. Variable $\delta m$ is an order of magnitude multiplication factor. For simplicity, we refer to Eq. (17) as an augmented or perturbed objective function.

\subsection{Statistical Uncertainty in Random Variable Parameters}

One type of epistemic uncertainty is the so-called statistical uncertainty, which is related to the limited number of samples that is used to characterize the probability distribution and the parameters of the random variables/processes describing intrinsic uncertainties. Statistical uncertainty can be reduced by increasing the number of samples and it could, theoretically, be eliminated by using infinite samples. Neither is the case for real world applications, where the number of samples is limited by budgetary constraints. Hence, statistical uncertainty is always present, in smaller or greater amounts.

There are many statistical model fitting approaches that allow statistical uncertainties in model parameters (mean, standard deviation) to be quantified probabilistically [33]. Since this paper addresses a generic problem, it is assumed that no probabilistic description of model parameters is available. The fundamental risk optimization problem considered herein is insensitive to a translation of the means $\left(\mu_{R}\right.$ and $\mu_{S}$ ), although not insensitive to a perturbation of the means. However, perturbation of the means is not considered in Eq. (17) . Nevertheless, a perturbation of the c.o.v. $(\rho)$ is assumed to reflect the uncertainty in the standard deviation of both $R$ and $S$ random variables.

Figure 3 illustrates the isolated effect of perturbations $\delta \rho=0 \pm 0.2$ in the objective function [Eq. (17)] of the fundamental risk optimization problem (for $\delta m=\delta k=0$ ), for $\rho=0.1$ and $\rho=0.3$ and for different cost multipliers $k$. It can be observed in Figure 3 that the effect of such perturbations is different for the optimum value $\lambda_{p}^{*}$ and for the cost function $\mathrm{J}_{p}\left(\lambda_{p}^{*}\right)$. For $\rho=0.1$, for example, the change in $\lambda_{p}^{*}$ is relevant, but the change in $\mathrm{J}_{p}\left(\lambda_{p}^{*}\right)$ is much smaller. The change in objective function $\mathrm{J}_{p}\left(\lambda^{*}\right)$ is actually more relevant for the present investigation. The dotted vertical lines in Fig. 3 indicate the change in perturbed cost functions for the optimum solution $\lambda^{*}$ of the original, unperturbed problem. The optimum solution $\lambda^{*}$ is found for the original problem, but the actual cost could be any of the perturbed costs $\mathbf{J}_{p}\left(\lambda^{*}\right)$. In Fig. 3 it can be observed that the largest effects of perturbations $\delta \rho=0 \pm 0.2$ occur for $\rho=0.3$ and $k=20$ (bottom right). In Figure 3, the curves leading to larger costs are obtained for $\mathrm{J}_{p}\left(\lambda^{*}, \delta \rho=+0.2\right)$, suggesting that actual design costs could likely be larger than the nominal, predicted cost $\mathrm{J}\left(\lambda^{*}\right)$. The curves leading to smaller costs are obtained for $\mathrm{J}_{p}\left(\lambda^{*}, \delta \rho=-0.2\right)$, where actual design costs are likely to be smaller than the predicted costs, but where the possibility for even further improvements is lost.

One kind of statistical uncertainty relates to the choice of probability distribution function (PDF) used to describe a given set of experimental observations. Hypothesis testing and distinct fitting tests [chi-square, Kolmogorov Smirnoff (KS), Anderson-Darling] can be used, but no unique PDF can ever be identified. Usually, a number of different PDFs will pass the fitting tests and could be deemed acceptable to represent the same set of data. However, the failure probability [Eq. (3)], is known to be highly sensitive to the tails of the assumed distributions. Hence, one significant source of epistemic uncertainty is the arbitrary choice between different acceptable PDFs. The simplified risk optimization problem presented herein is not appropriate for an investigation of this uncertainty because a closed-form solution is only available for Gaussian distributions. Hence, probability distribution uncertainties are not considered. In the 

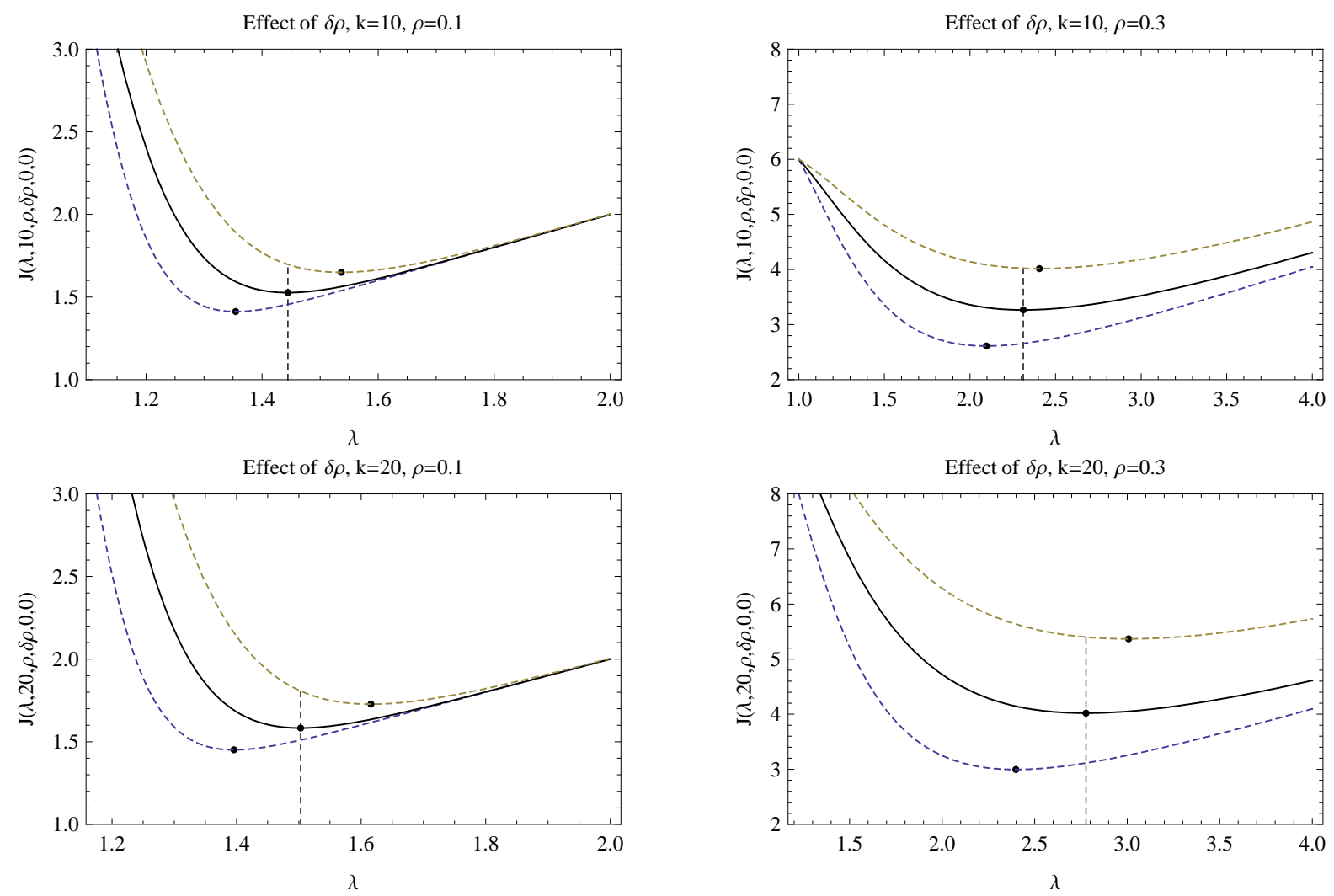

FIG. 3: Effect of perturbations $\delta \rho= \pm 0.2$ in cost functions and $\lambda^{*}$.

application example presented later, different PDFs are considered. In a future extension of the present investigation, the principle of maximum entropy will be used to more comprehensively investigate the effects of uncertainty in probability distribution models.

\subsection{Uncertainty in Failure Costs}

This uncertainty relates to the deterministic unpredictability of (future) failure costs. In real engineering structures, such uncertainties arise, for example, from fluctuations in the price of commodities, since failure costs are always paid in the future. For failures involving human injury or death, or environmental damage, failure costs can always be quantified (from the practical managers point of view, at least) from the amount of past compensation payoffs for similar accidents. Clearly, such values cannot be defined with certainty. For the general risk optimization problem considered herein, these uncertainties cannot be quantified. However, an order of magnitude interval can be determined based on intuition. Figure 4 illustrates the effects of isolated perturbations $\delta k=0 \pm 0.2$ in objective function [Eq. (17)], for $\delta \rho=\delta m=0$. It is observed that a perturbation of the same order (as $\delta \rho=0 \pm 0.2$ ) produces much smaller effects for optimum points $\left(\lambda_{p}^{*}\right)$ and for perturbed cost functions, $\mathbf{J}_{p}\left(\lambda^{*}\right)$. Moreover, it also can be observed that the largest effects of perturbations $\delta k=0 \pm 0.2$ occur for $\rho=0.3$ (Fig. 4, right).

\subsection{Model Errors and Phenomenological Uncertainty}

Model errors arise from the inability of structural load and resistance models to exactly predict loads, load effects, and resistance of structural members or systems. In civil engineering design it is customary to formulate limit state functions in terms of load effects and resistance of structural members. A resistance model gives the resistance of a 

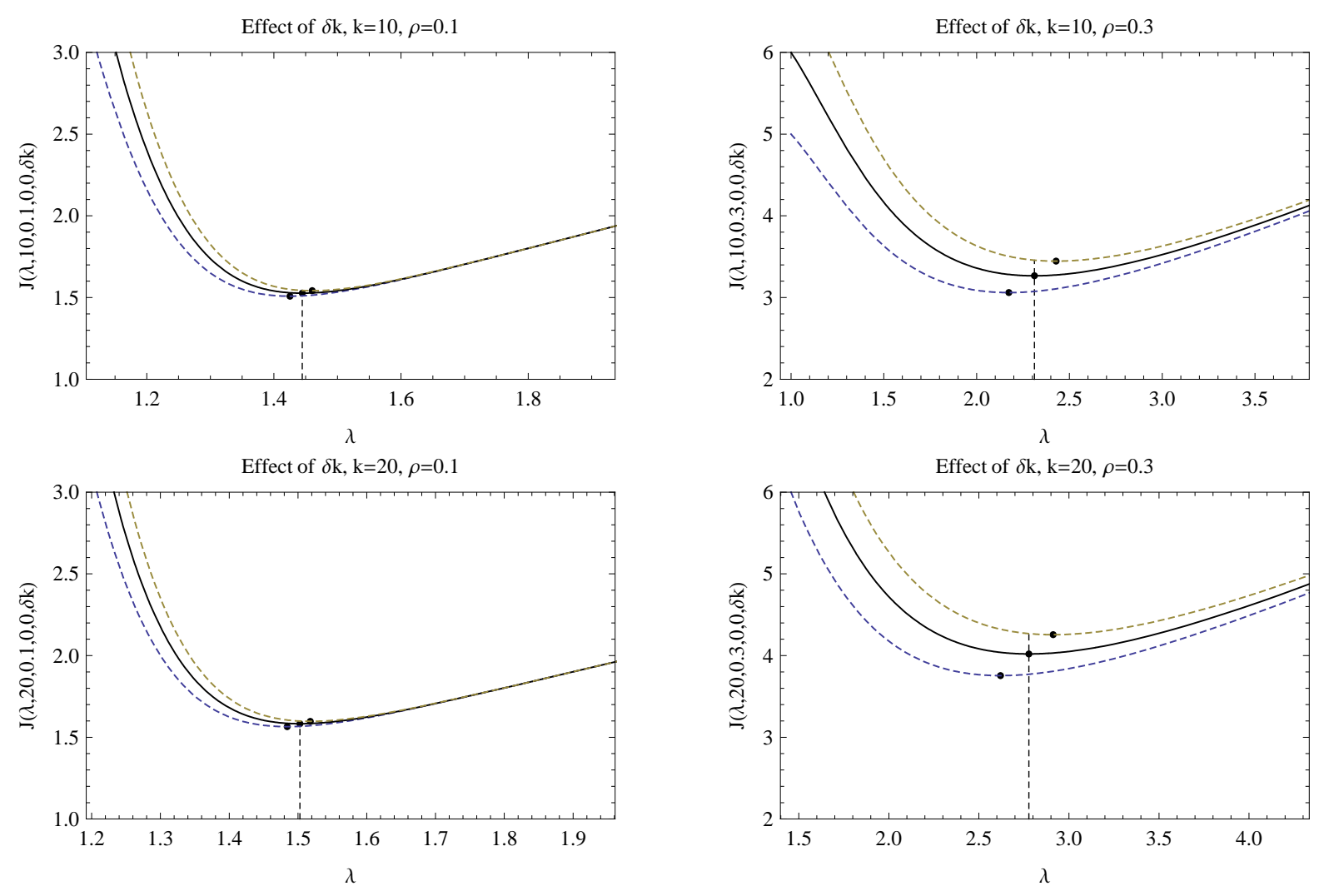

FIG. 4: Effect of perturbations $\delta k=0 \pm 0.2$ in cost functions and $\lambda^{*}$.

structural member in terms of member dimensions and resistance of constituting structural materials. The resistance of a reinforced concrete element, for example, is a function of reinforcement steel yield stress $\left(\sigma_{s}\right)$ and bar areas $\left(A_{s}\right)$, concrete strength and area $\left(\sigma_{c}, A_{c}\right)$, cross-section dimensions $(B, H)$, and other relevant variables:

$$
R^{\text {model }}=R\left(\sigma_{c}, \sigma_{s}, A_{c}, A_{s}, B, H, \ldots\right) .
$$

Many model errors can be described probabilistically by comparing model predictions with experimental results $[34,35]$. In the example above, model error samples can be obtained from experimental results where the model parameters are varied:

$$
M^{\text {error }}=\frac{R^{\text {experimental }}}{R^{\text {model }}} .
$$

When experimental results are available, model error can be represented by a proper PDF and incorporated into the formulation, becoming a new but probabilistically quantifiable source of uncertainty. The new resistance estimate, incorporating model uncertainties, becomes

$$
R=M^{\text {error }} R^{\text {model }} .
$$

It is assumed herein that such quantifiable model error uncertainties have already been incorporated in the load $(S)$ and resistance $(R)$ variables in the limit state equation [Eq. (7)]. However, model error uncertainties are not always quantifiable. Additional uncertainties exist. For example, the actual resistance of a structural element, as constructed and in service condition, cannot be captured by laboratory experiments. Manufacturing and actual service conditions (boundary conditions, loading positioning and orientation, etc.) of the element cannot be predicted exactly. Hence, there are always additional sources of model uncertainties that cannot be quantified probabilistically. In this paper, interest lies in the effect of these epistemic uncertainties on the nominal, calculated failure probabilities [Eqs. (3) and 
(9)]. To take into account epistemic uncertainties that directly affect the nominal, calculated failure probabilities, the perturbation $\delta m$ was included in Eq. (17). One such epistemic uncertainty is the probability distribution model for random variables $R$ and $S$, as mentioned before.

One large source of uncertainty for most engineering design problems is of phenomenological origin. Phenomenological uncertainty describes uncertainty related to the analyst's understanding of the phenomena that actually control the behavior of the systems he designs. One typical example is structural failure that occurs under failure modes that, for the original designer of the structure, is unpredictable or unimaginable. The history of structural engineering has many examples of such failures. Dynamic wind excitation, which led to the collapse of the Tacoma Narrows Strait bridge, was not known or not regarded as a relevant failure mode for which bridges had to be designed against in past years. In another example, the World Trade Center in New York had been designed to withstand the collapse of a small aircraft, and a small fire fueled by office material-like paper, carpets, timber boards, and so on. A fire of enormous intensity, fueled by large amounts of jet fuel, had not been anticipated by the designers, and ultimately led to the collapse of the towers.

Phenomenological uncertainty can be very large, and can affect the nominal, calculated, failure probabilities by orders of magnitude. Non-quantified model errors and distribution model uncertainties will add to the uncertainty affecting calculated failure probabilities. In order to study the effects of epistemic uncertainties on calculated failure probabilities, the order of magnitude perturbation $\delta m$ was introduced in Eq. (17).

Figure 5 illustrates the effects of isolated perturbations $\delta m=0 \pm 1$ in objective function [Eq. (17)], for $\delta \rho=\delta k=$ 0 . This perturbation of \pm 1 order of magnitude $\left(10^{-1}\right.$ and $\left.10^{+1}\right)$ is considered representative of the combined effects of phenomenological, model error, and distribution model uncertainties. It is observed in Fig. 5 that such perturbations change the risk optimization objective function quite dramatically. For $\rho=0.1$ the change in $\lambda_{p}^{*}$ is not too large, but the change in objective function $\mathrm{J}_{p}\left(\lambda^{*}\right)$ is already significant. For $\rho=0.3$ both $\lambda_{p}^{*}$ and $\mathrm{J}_{p}\left(\lambda^{*}\right)$ vary significantly. The
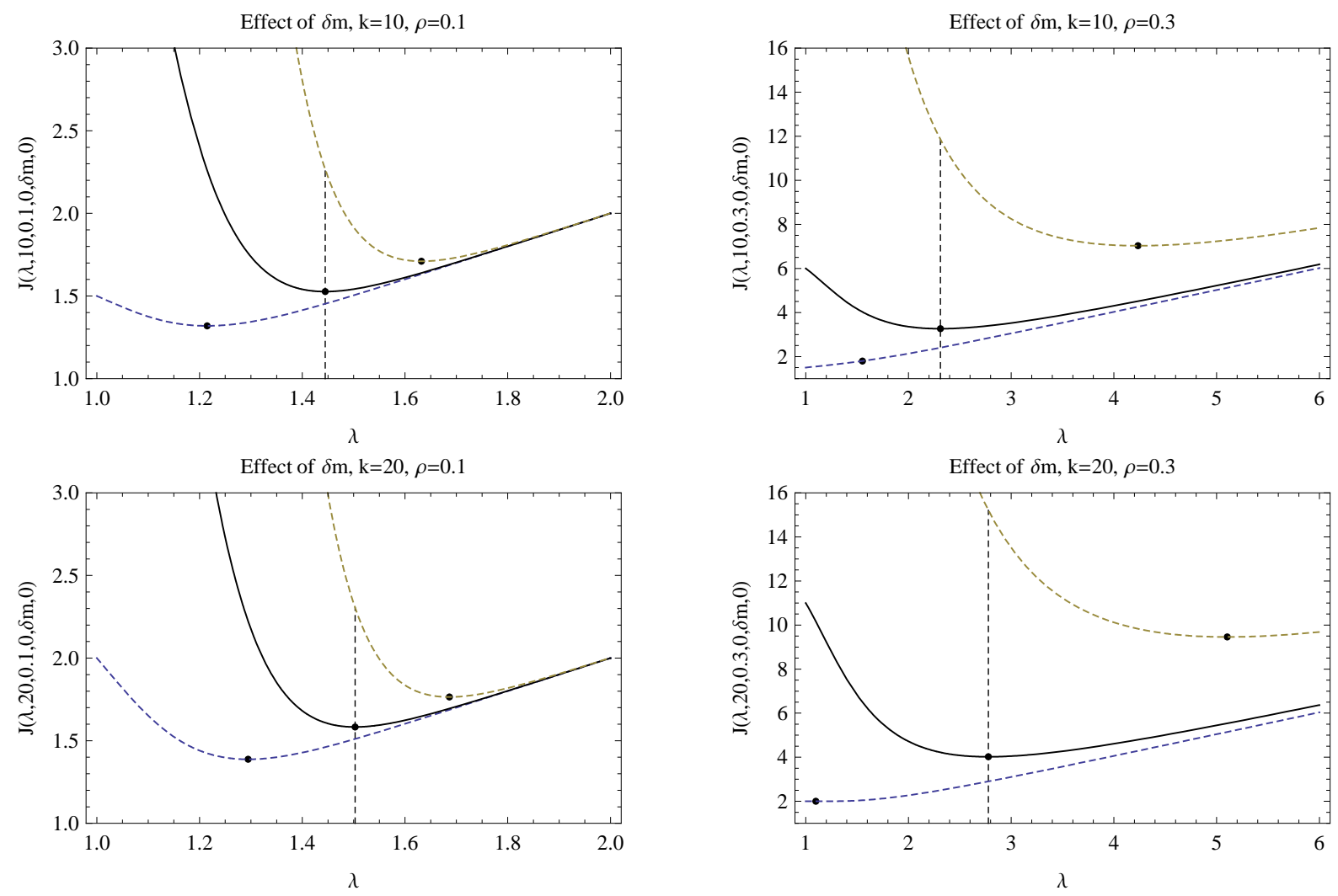

FIG. 5: Effect of perturbations $\delta m=0 \pm 1$ in cost functions and $\lambda^{*}$.

Volume 2, Number 1, 2012 
change in $\mathrm{J}_{p}\left(\lambda^{*}\right)$ is more relevant for the perturbation $\delta m=+1$, since this increases the nominal, calculated failure probability by a factor of 10 , largely increasing expected costs of failure. For perturbation $\delta m=-1$, the cost $\mathbf{J}_{p}\left(\lambda^{*}\right)$ is actually smaller than $\mathrm{J}\left(\lambda^{*}\right)$, and also not much different than $\mathrm{J}\left(\lambda^{*}\right)$.

It is clear from Figs. 3-5 that perturbations $\delta m=0 \pm 1$ have greater impact than $\delta \rho=0 \pm 0.2$ or $\delta k=0 \pm 0.2$. However, the combined effects of these perturbations can have an even greater impact on results of risk optimization.

\subsection{Effect of Combined Uncertainties}

The individual uncertainties represented by $\delta \rho, \delta k$, and $\delta m$, studied in this section, illustrate the fundamental risk optimization problem's sensitivity to different types of epistemic uncertainties. Considering the combined effect of these (interval) uncertainties would represent a worst-case, unlikely scenario. Hence, in order to investigate the combined effect of these uncertainties and in order to formulate a robust version of the risk optimization problems, a fuzzy description of epistemic uncertainities is considered.

\section{FUZZY REPRESENTATION OF EPISTEMIC UNCERTAINTIES}

A fuzzy-variable description is admitted for the quantification of epistemic uncertainties affecting risk optimization problems. A fuzzy variable with triangular (linear) shape can be represented as [5, 32]

$$
z=\operatorname{tri}\left(z_{i}, \bar{z}, z_{f}\right)
$$

where $\bar{z}$ is the so-called center, nominal, or crisp value, and $\left[z_{i}, z_{f}\right]$ is the interval of definition. For a triangular fuzzy variable, the following membership function $m_{z}(z) \in[0,1]$ is defined:

$$
m_{z}(z)=\min \left[\max \left(0,1-\frac{\bar{z}-z}{\bar{z}-z_{i}}\right), \max \left(0,1-\frac{z-\bar{z}}{z_{f}-\bar{z}}\right)\right], \quad \forall z \in \mathbb{R} .
$$

Other types of fuzzy variables are available, but the triangular shape is adequate for the present analysis. Figure 6 illustrates the membership function for a variable $z=\operatorname{tri}(0.8,1.0,1.2)$, which is used to represent the uncertainties in $\rho$ and $k$. A similar representation is used for the uncertainty in the calculated failure probabilities, $\delta m$ :

$$
\begin{gathered}
\rho=\bar{\rho} \operatorname{tri}(0.8,1.0,1.2), \\
k=\bar{k} \operatorname{tri}(0.8,1.0,1.2), \\
\delta m=\operatorname{tri}(-1,0,+1) .
\end{gathered}
$$

The membership function is equal to one for the center value, $m_{z}(\bar{z})=1$. For decreasing values of $m_{z}$, nested intervals are obtained. Figure 6 (right) illustrates the computation of intervals for fuzzy variable $z=\operatorname{tri}(0.8,1.0,1.2)$ and for $m_{z}=0.2$.

Using a fuzzy representation of the epistemic uncertainties [Eq. (23)], fuzzy costs $\mathbf{J}^{\text {fuzzy }}(\lambda, k, \rho)$ are computed. For discrete values of the membership function $\left(m_{z}=0.0\right.$ to $m_{z}=0.9$, with steps of 0.1$)$, intervals are computed using the minimum and maximum values of each of the triangular fuzzy variables. This can be done because the cost function is monotonically increasing with the parameters. For all combinations of the three perturbations, the largest and smallest perturbed costs $\mathbf{J}_{p}\left(\lambda^{*}\right)$ are evaluated [Eq. (17)], yielding the corresponding bounds of the fuzzy cost for that membership level. Figure 7 illustrates the fuzzy costs, in continuous lines, for different problem parameters ( $\rho=0.1$ and $\rho=0.3 ; k=10$ and $k=20$ ). It can be observed that fuzzy costs are skewed toward larger, positive values, originated from perturbations leading to larger failure probabilities $(\delta \rho=+0.2$ and $\delta m=+1)$ and larger failure costs $(\delta k=+0.2)$, as shown in Figures 3-5.

\section{ROBUST RISK OPTIMIZATION}

Using a fuzzy representation of epistemic uncertainties, fuzzy cost functions are evaluated (following Section 4) for each problem configuration. A robust cost function, $\mathrm{J}_{R}(\lambda, k, \rho)$, is introduced: 

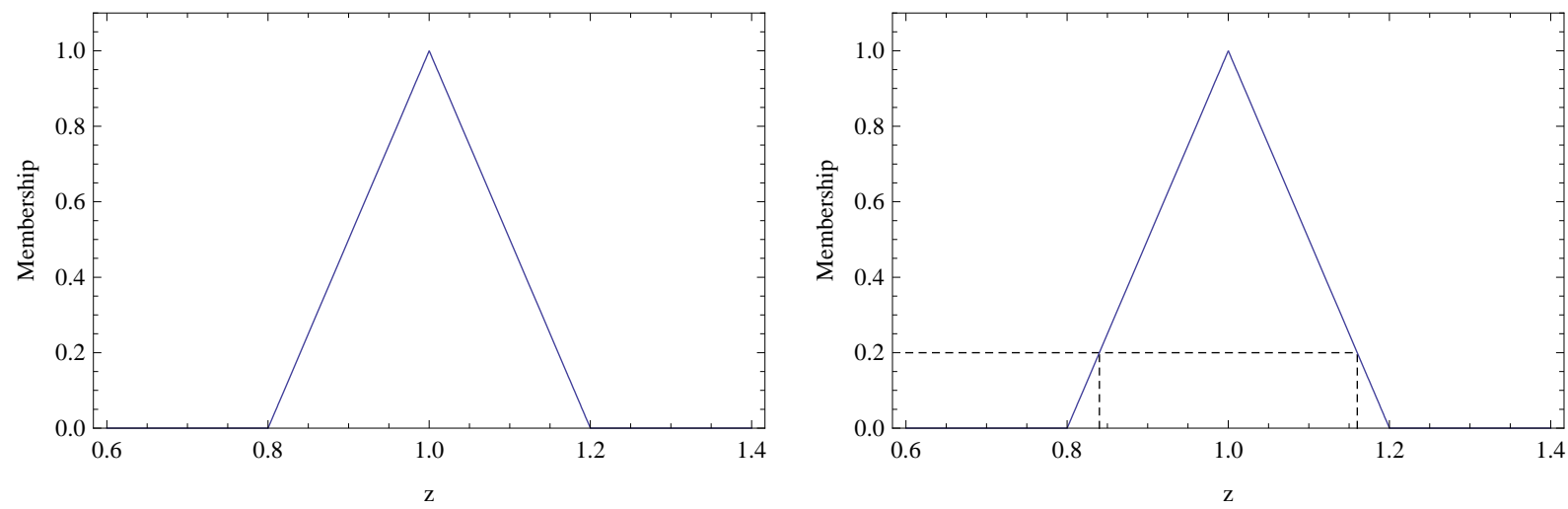

FIG. 6: Membership function (left) for fuzzy representation of variable $z=\operatorname{tri}(0.8,1.0,1.2)$ and determination of interval corresponding to membership level $m_{z}=0.2$ (right).
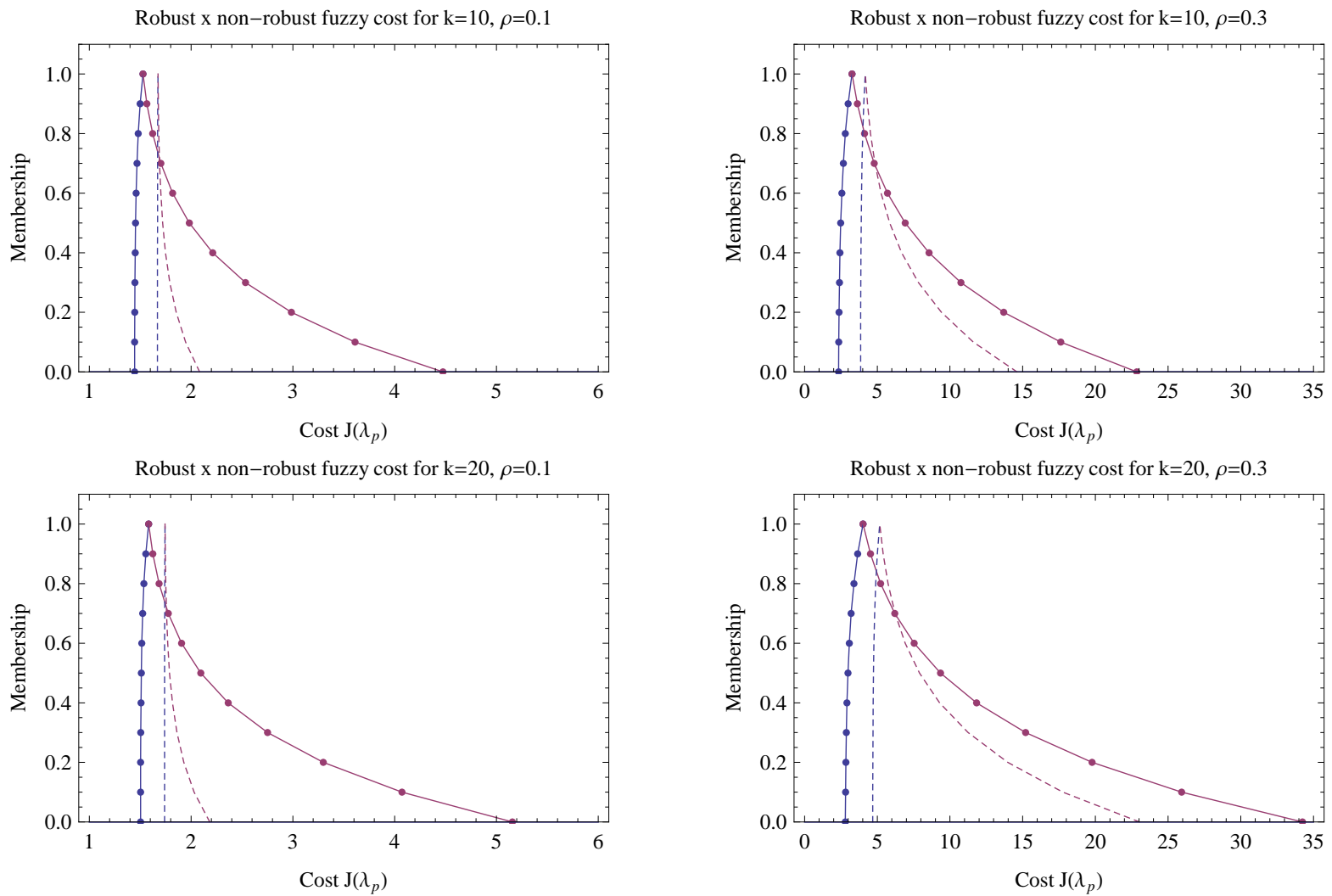

FIG. 7: Robust and non-robust fuzzy costs in terms of parameters $k$ and $\rho$ for combined effect of fuzzy uncertainties.

$$
\mathbf{J}_{R}(\lambda, k, \rho)=\overline{\mathbf{J}}^{\text {fuzzy }}(\lambda, k, \rho)+\int_{\mathbf{J}_{i}}^{\mathbf{J}_{f}} m_{\mathbf{J}^{\text {fuzzy }}}(x) d x,
$$

using the notation introduced in Eqs. (22) and (23), and where $\bar{J}^{\text {fuzzy }}(\lambda, k, \rho)$ is the crisp value and $m_{\text {fuzzy }}(x)$ is the membership function of the fuzzy cost (Fig. 7). Equation (24) integrates the whole portfolio of fuzzy costs due to the epistemic uncertainties. If the fuzzy cost function could be made equal to the nominal cost, for example, by reducing the distance $\mathbf{J}_{f}-\mathrm{J}_{i}$ to zero, then the integral in Eq. (24) would reduce to zero and the robust cost would be equal to the 
nominal cost. In the proposed robust risk optimization, one looks for the central safety factor $\lambda_{R}$, which minimizes (or which is robust with respect to) the whole portfolio of fuzzy uncertainties:

$$
\lambda_{R}=\underset{\lambda}{\arg \min }\left\{\mathbf{J}_{R}(\lambda, k, \rho): \lambda>1\right\} .
$$

Results are shown in Fig. 8, where nominal and fuzzy cost functions are compared and where the nominal $\left(\lambda_{0}\right)$ and robust $\left(\lambda_{R}\right)$ optima are identified. The fuzzy cost function $\mathrm{J}_{R}$ is clearly more expensive than the non-robust counterpart, as it incorporates all the fuzzy costs. As a consequence, the robust optima $\left(\lambda_{R}\right)$ are more conservative then the nonrobust optima. The differences between robust and non-robust optima and cost functions are more pronounced for higher levels of uncertainty ( $\rho=0.3$ in comparison to $\rho=0.1$ ) of the underlying reliability problem. Differences also increase for higher failure costs $(k=20$ in comparison to $k=10)$.

In Fig. 7, the robust and non-robust fuzzy cost functions are compared. The continuous lines illustrate the fuzzy costs [ $\left.\mathrm{J}^{\mathrm{fuzzy}}\left(\lambda_{0}, k, \rho\right)\right]$ for the original, non-robust optimum solutions $\lambda_{0}$. The dashed lines show the same fuzzy costs for the robust solution, $\mathrm{J}^{\mathrm{fuzzy}}\left(\lambda_{R}, k, \rho\right)$. It can be seen in Fig. 7 that the robust formulation has led to solutions that are clearly less sensitive to the fuzzy epistemic uncertainties.

\section{APPLICATION EXAMPLE: CODIFIED DESIGN OF STEEL MEMBER IN BENDING}

A simple design-code application example is considered in this section. Following the American Society of Civil Engineers (ASCE) standard [36], the design equation for a structural member subject to dead and live loads is given by
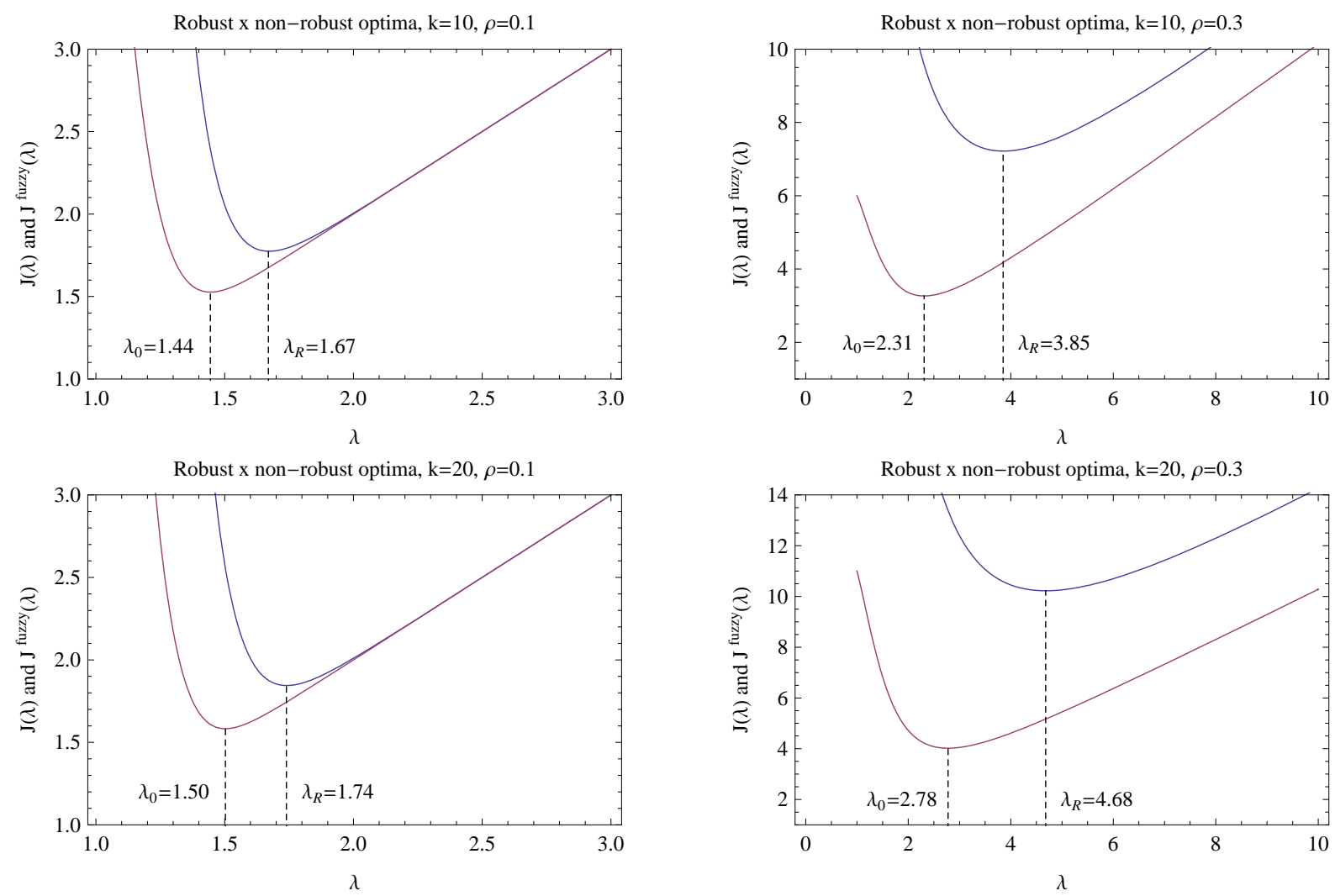

FIG. 8: Robust and non-robust cost functions and optima in terms of parameters $k$ and $\rho$ for combined effect of fuzzy uncertainties. 


$$
\begin{gathered}
R_{D} \geq S_{D}, \\
\phi_{R} R_{n} \geq \gamma_{D} D_{n}+\gamma_{L} L_{n},
\end{gathered}
$$

where $R_{D}$ and $S_{D}$ are the design values of resistance and load, respectively; $\gamma$ are partial safety factors applied on (nominal values of) loads; $D_{n}$ is the nominal dead load; $L_{n}$ is the nominal live load; $R_{n}$ is a nominal member resistance; and $\phi_{R}$ is a partial safety factor applied on nominal member resistance. Following [36], in a conventional design situation, $\gamma_{D}=1.2$ and $\gamma_{L}=1.6$. Following [37], in the design of steel members in bending, $\phi_{R}=0.9$. Introducing an extra safety margin $(\lambda>0)$ to account for failure consequences, and replacing the inequality by an equality, one obtains

$$
\phi_{R} R_{n}=\lambda\left(\gamma_{D} D_{n}+\gamma_{L} L_{n}\right)
$$

For a given nominal resistance and for a specified load ratio $\left(L_{n} / D_{n}\right)$, the nominal dead load is obtained as

$$
D_{n}=\frac{1}{\lambda} \frac{\phi_{R} R_{n}}{\left(\gamma_{D}+\gamma_{L} L_{n} / D_{n}\right)} .
$$

The nominal live load is evaluated accordingly. The limit state equation for this problem is

$$
g(\lambda, \mathbf{X})=R-(D+L)=0,
$$

where $R, D$, and $L$ are the random variable resistance, dead, and live loads, respectively. This is an implicit function of $\lambda$, since the nominal loads are functions of $\lambda$ [Eq. (28)].

Parameters of the problem's random variables are evaluated from the data in Table 1, following Ellingwood and Galambos [38]. Table 1 presents nominal values of the distribution parameters (mean and c.o.v.) and probability distributions that were used by Ellingwood and Galambos [38] in the calibration of partial safety factors for the American design codes $[36,37]$. In the present application example, the optimum safety margin $\lambda^{*}$ for different failure costs is considered, and made robust with respect to epistemic uncertainties affecting the parameters in Table 1.

The initial or construction cost is assumed proportional to $\lambda$, and the cost of failure is $k \lambda$, where $k=5$ and $k=20$ are considered. Following [30], $k=5$ corresponds to a more conventional design situation, whereas $k=20$ represents an extreme situation. Hence, the objective function for non-robust optimization is:

$$
\mathbf{J}^{\text {total }}(\lambda, k)=\mathbf{J}(\lambda, k)=\mathbf{J}^{\text {initial }}(\lambda)+\mathbf{J}^{\text {expected }}(\lambda, k)=\lambda+k \lambda P_{f}(\lambda) .
$$

The failure probability is evaluated using Eqs. (3) and (29), through the FORM [25]. For the robust optimization, the objective function is given by Eq. (24).

Following the discussion in Section 3, uncertainties of epistemic nature are considered to exist with respect to the mean values, c.o.v. and probability distributions presented in Table 1. Perturbations of $\delta \mu=0.05$ and $\delta \rho=0.05$ are considered, for the means and c.o.v. ( $\rho$ ) given in Table 1. Perturbations of the failure cost terms in Eq. (30) are also considered: $\delta k=0.10$. These uncertainties are represented as triangular fuzzy variables, following Section 4:

$$
\begin{gathered}
\mu=\bar{\mu} \operatorname{tri}(1-\delta, 1.00,1+\delta), \\
\rho=\bar{\rho} \operatorname{tri}(1-\delta, 1.00,1+\delta), \\
k=\bar{k} \operatorname{tri}(1-2 \delta, 1.00,1+2 \delta), \delta=0.05 .
\end{gathered}
$$

With two levels of uncertainties (positive and negative perturbations) in two parameters ( $\mu$ and $\rho$ ) of three random variables, a total of 12 combinations results. These have to be evaluated for different membership levels and also

TABLE 1: Nominal parameters and distributions of load and resistance variables

\begin{tabular}{|c|c|c|c|}
\hline Variable & Mean $(\bar{\mu})$ & c.o.v. $(\bar{\rho})$ & Distribution \\
\hline Resistance of steel member in bending & $1.18 R_{n}$ & 0.15 & Lognormal (LN) \\
Dead load & $1.05 D_{n}$ & 0.10 & Normal (N) \\
Live load & $1.00 L_{n}$ & 0.25 & Gumbel (G) \\
\hline
\end{tabular}

Volume 2, Number 1, 2012 
combined with the perturbations PDFs, to be described. This could represent a significant computational burden for practical (numerically intensive) application problems. Fortunately, not all possible combinations have to be considered. Although the cost function does not vary monotonically with the failure probability, limiting values of the fuzzy cost (lower and upper bounds for each membership level) originate in the smallest and largest failure probabilities. Hence, only those combinations knowingly leading to the smallest and largest failure probabilities need to be considered. In the present example, this represents a positive perturbation in the mean resistance $\left[\mu_{R}=\bar{\mu}_{R}(1+\delta \mu)\right]$, combined with a negative perturbation in both mean loads $\left[\mu_{D}=\bar{\mu}_{D}(1-\delta \mu)\right.$ and $\left.\mu_{L}=\bar{\mu}_{L}(1-\delta \mu)\right]$, and combined with a negative perturbation of the c.o.v. for the three variables, which leads to the smallest failure probabilities. Conversely, a negative perturbation in mean resistance, with positive perturbations in mean loads and positive perturbation in c.o.v. $[\rho=\bar{\rho}(1+\delta \rho)]$, leads to the largest failure probabilities. Hence, only two combinations have to be considered instead of 12 .

Epistemic uncertainties in the probability distributions are also considered in this application example. Such discrete uncertainties are difficult to model using fuzzy variables; hence, only a few combinations of different (possible) (PDFs) are considered. The nominal PDFs are those presented in Table 1 and used by Ellingwood and Galambos [38] in the calibration of ASCE design codes. However, it is admitted that the resistance can be represented as well by a normal $(\mathrm{N})$ distribution with the same mean and standard deviation. Similarly, it is admitted that the dead load could be modeled using a log-normal (LN) distribution. For the live load, whose uncertainty is known to dominate this problem, three possible extreme-value distributions are considered: Gumbel, Frechet, and Weibull. In this case, there is no guarantee that some combinations of PDFs would lead to smallest or largest failure probabilities; hence, all combinations would have to be considered. However, considering the illustrative purpose of this example, only four combinations are considered herein:

$$
\begin{aligned}
\{R, D, L\} & \sim\{\mathrm{LN}, \mathrm{N}, \text { Gumbel }\} \text { "nominal PDFs", } \\
& \sim\{\mathrm{LN}, \mathrm{N}, \text { Frechet }\} \\
& \sim\{\mathrm{LN}, \mathrm{N}, \text { Weibull }\} \\
& \sim\{\mathrm{N}, \mathrm{LN}, \text { Gumbel }\}
\end{aligned}
$$

Figure 9 illustrates the differences between these probability distributions for $R_{n}=10$ units and for load ratio $L_{n} / D_{n}=2$. It is observed that the main difference between these distribution models is for live load $L$ and for resistance $R$.

The numerical examples presented in this article are computed in an algorithm implemented in the Mathematica 6.0 software. Illustrations are generated by the same algorithm.

In the computation of the nominal fuzzy cost (membership level $m_{z}=1$ ), only the nominal probability distributions are considered. However, in the computation of fuzzy costs bounds (smallest and largest values) for other membership levels, the four admitted PDF combinations are considered. Figure 10 illustrates the resulting fuzzy costs, and compares fuzzy costs with and without the PDF perturbations. It can be observed that the effect of PDF perturbations is a moderate increase in fuzzy cost uncertainty. The effect of PDF perturbations is more pronounced in terms of reducing the expected costs for higher membership levels (smaller perturbations in other problem parameters).

Nominal costs and fuzzy costs are compared in Fig. 11, which also identifies the nominal optimum $\left(\lambda_{0}\right)$ and robust optimum $\left(\lambda_{R}\right)$ solutions for this problem. Essentially, the behavior is similar to what was observed for the fundamental risk optimization problem (Fig. 8): fuzzy cost functions are higher (more expensive) than their nominal counterparts, and robust optima are more conservative than their nominal, non-robust counterparts. Also, the nominal cost for the robust optimum, $\mathrm{J}\left(\lambda_{R}, k\right)$, is higher than the nominal cost for the nominal, non-robust solution:

$$
\mathrm{J}\left(\lambda_{R}, k\right)>\mathbf{J}\left(\lambda_{0}, k\right) .
$$

Fuzzy costs for the robust and non-robust solutions are compared in Fig. 12. It can be observed that the robust formulation leads to larger nominal costs, but to fuzzy costs that are less sensitive (reduced area) with respect to fuzzy epistemic uncertainties. 


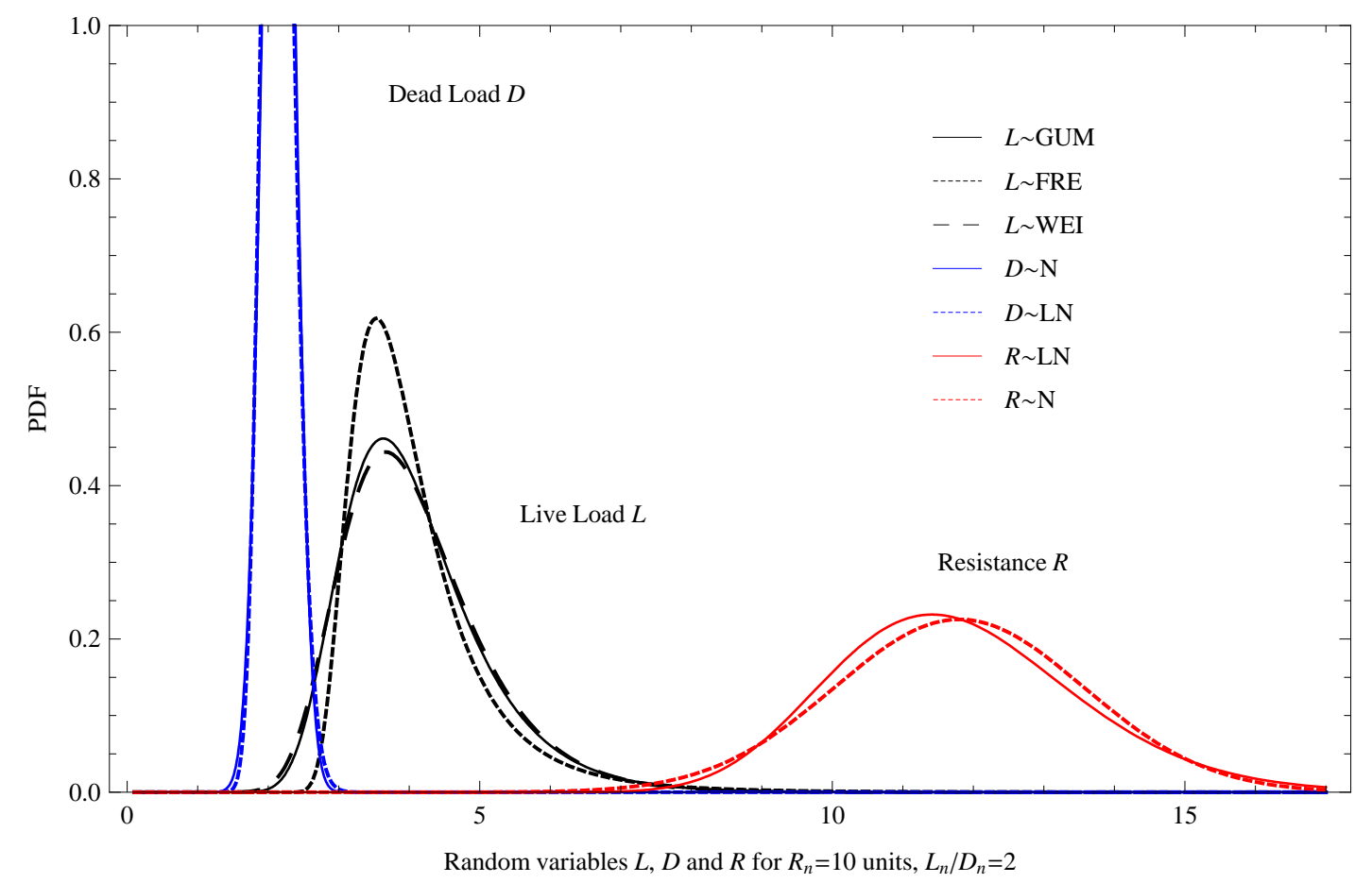

FIG. 9: Comparison of probability distribution functions considered in the application example.
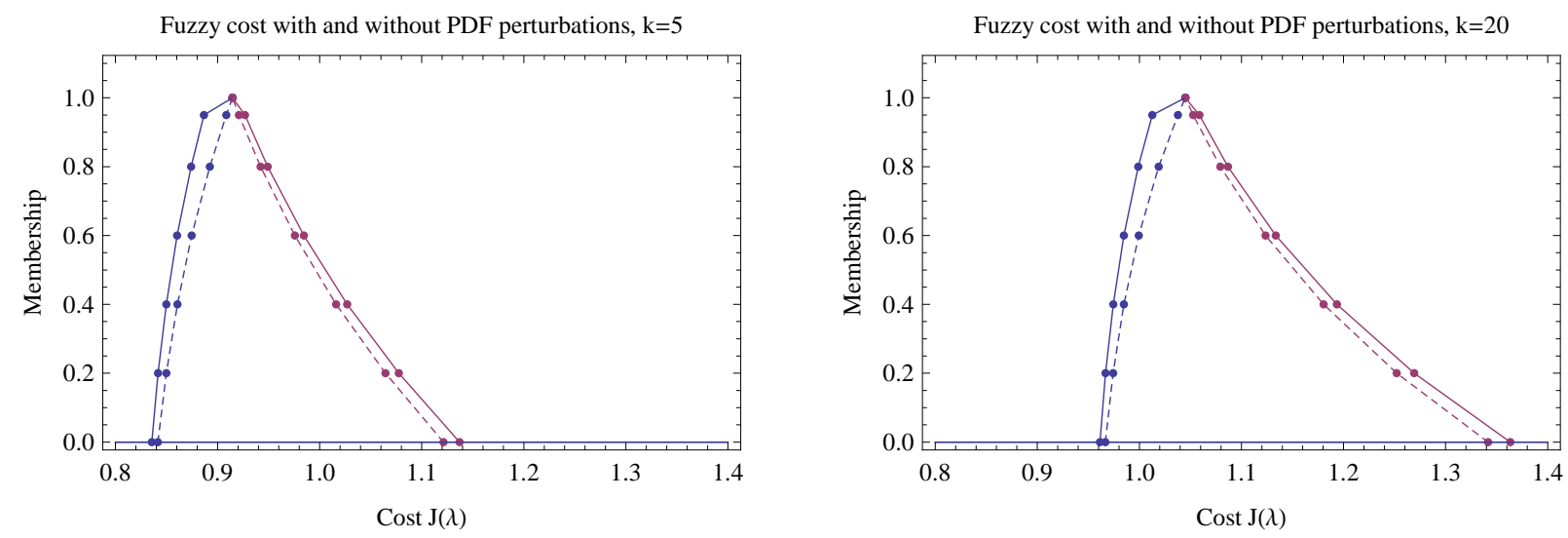

FIG. 10: Fuzzy costs with (continuous line) and without (dashed line) probability distribution function perturbations.

For the present application problem, the actual values of the obtained optima also are relevant. For instance, for $k=5$ both non-robust $\left(\lambda_{0}=0.82\right)$ and robust $\left(\lambda_{R}=0.93\right)$ optima suggest that the current design condition $(\lambda=1.00)$ is slightly too conservative. However, one also observes that the current $\operatorname{cost} \mathrm{J}(\lambda=1.0 ; k=5.0)$ is not much larger than the optimum cost for $\mathrm{J}\left(\lambda_{R}=0.93 k=5.0\right)$. For $k=20$, the non-robust $\left(\lambda_{0}=0.95\right)$ and robust $\left(\lambda_{R}=1.07\right)$ optima are in reasonable agreement with the current professional practice $(\lambda=1.00)$. These results may not be simple coincidences: they actually suggest that some sort of (robust?) risk optimization has been performed in the past, even if not using explicit formulations like the one presented herein.

To a certain extent, the results presented above also depend on the load ratio $\left(L_{n} / D_{n}=2\right)$ considered. Robust $\left(\lambda_{R}\right)$ and non-robust $\left(\lambda_{0}\right)$ optima obtained for other load ratios, for $k=5$ and $k=20$, are presented in Fig. 13. Some change in the resulting optima can be observed but, in essence, the results are similar to the case for $L_{n} / D_{n}=2$. 

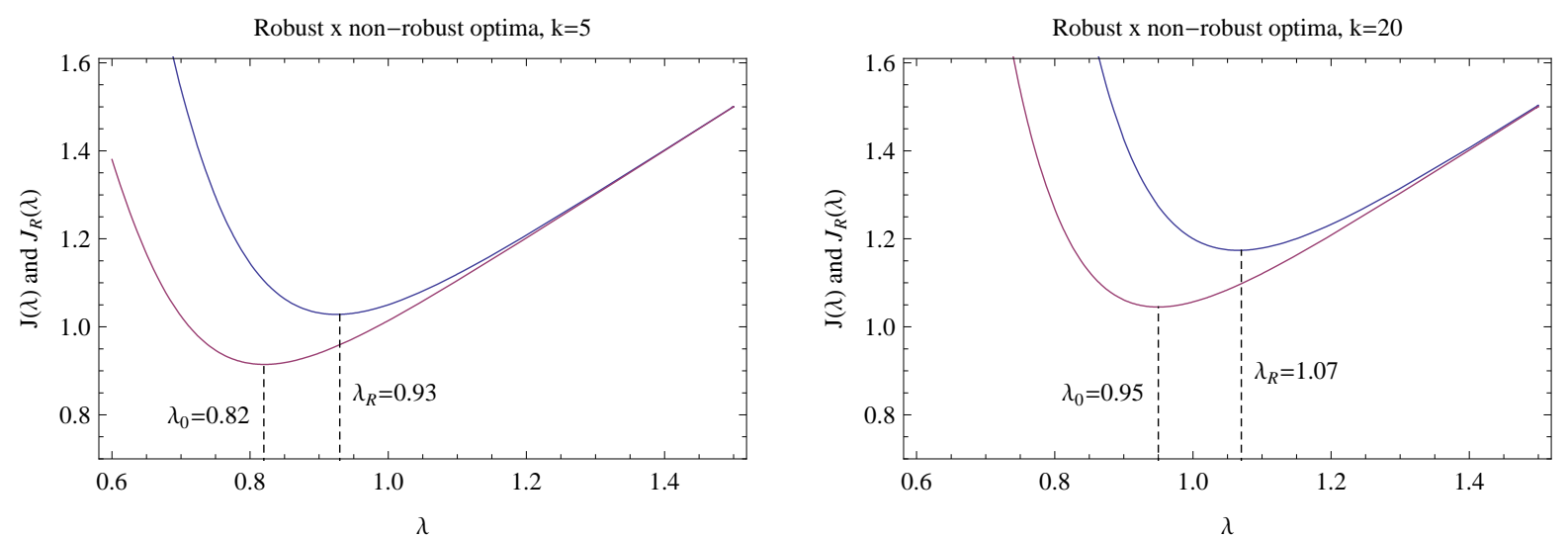

FIG. 11: Nominal and fuzzy cost functions, and corresponding nominal $\left(\lambda_{0}\right)$ and robust $\left(\lambda_{R}\right)$ optima for application problem.
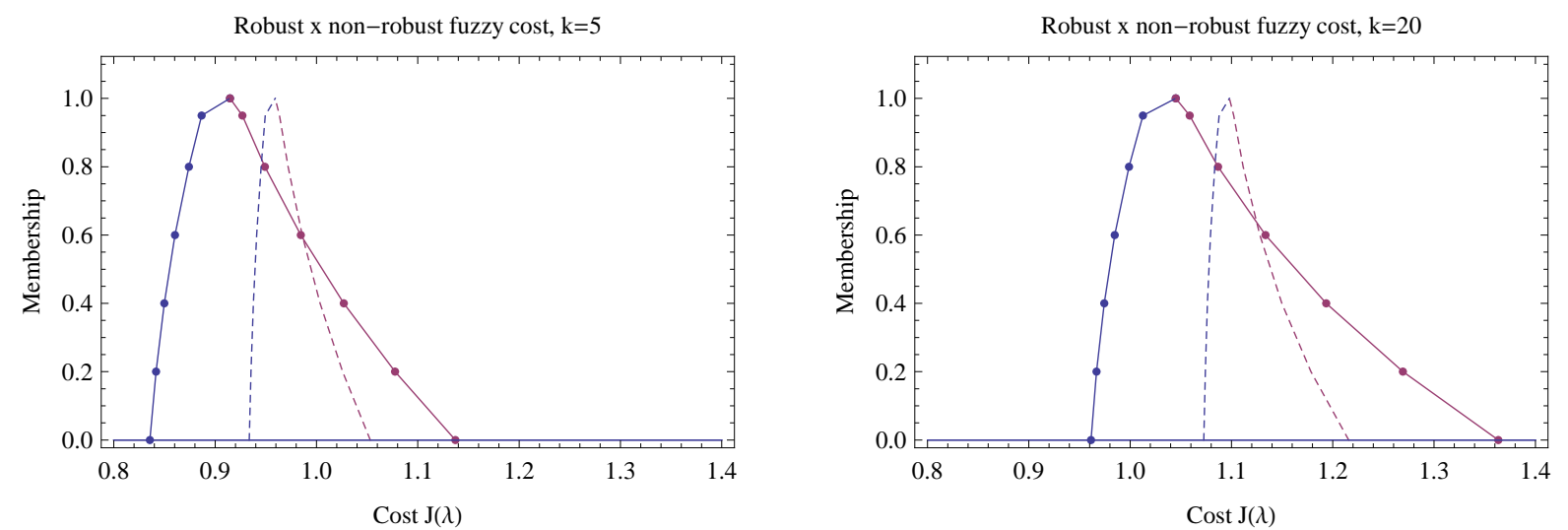

FIG. 12: Comparison of robust (dashed line) and non-robust (continuous line) fuzzy costs for application problem.
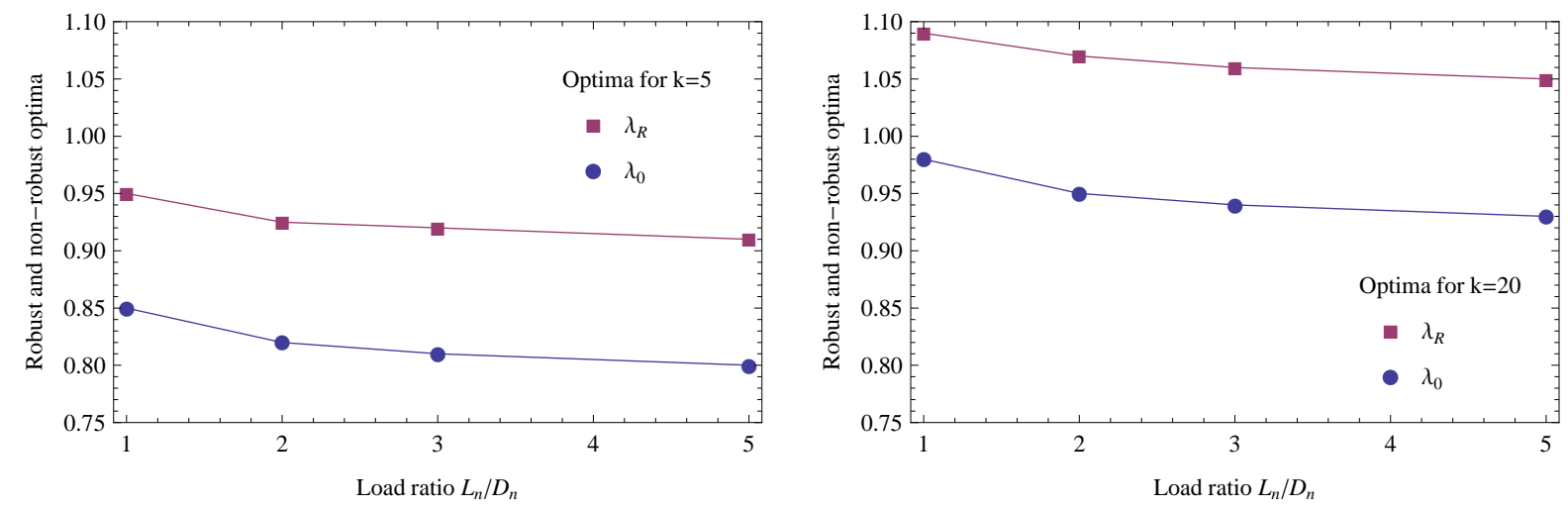

FIG. 13: Robust and non-robust optima for other load ratios $\left(L_{n} / D_{n}\right)$.

\section{EFFECT OF EXPERT CONFIDENCE}

Since the quantification of epistemic uncertainties is based on (individual) subjective judgment, it is clear that robust risk optimization, as presented herein, yields different results for different analysts. The effect of expert confidence in robust risk optimization results is investigated in this section, following the codified steel member design example. 
Hence, assume that the fuzzy representation of epistemic uncertainties in Eq. (31) corresponds to the judgment of a neutral expert $(\delta=0.05)$. Uncertainty modeling by a pessimistic expert is represented following Eq. (31), but with $\delta=0.10$. In contrast, for an optimistic expert $\delta=0.025$ is considered. In the limit with $\delta \rightarrow 0$, the (idealist) optimistic's result would correspond to $\lambda_{R}=\lambda_{0}$.

The codified steel member design example is solved again using the pessimistic and optimistic expert's representation of epistemic uncertainties, and also using some intermediate values. Results are presented in Fig. 14, in terms of the ratio $\lambda_{R} / \lambda_{0}$ of robust to non-robust optimum safety coefficients as well as the ratio of fuzzy costs for the nonrobust to robust coefficients, for $k=5$ and $k=20$. Is can be observed that these ratios are almost linearly dependent on the expert confidence factor $(\delta)$. Interestingly, as the expert becomes more pessimistic, his robust optimum $\left(\lambda_{R}\right)$ becomes more conservative, in comparison to the nominal, non-robust $\lambda_{0}$.

Robust and non-robust fuzzy cost functions are also compared, in Fig. 15, in terms of the pessimistic and optimistic modeling of epistemic uncertainties, for $k=5$ and $k=20$. It can be observed that the well-behaved differences in optimum $\lambda$ and fuzzy cost functions illustrated in Fig. 14 correspond to quite dramatic changes in the fuzzy cost functions.

Finally, it is observed that although results do dependent on the individual expert's quantification of epistemic uncertainties, the nature of these results remains unchanged: robust solutions are more conservative and more expensive, but less sensitive to epistemic uncertainties. The more pessimistic the expert, the more conservative are his results in comparison to nominal, non-robust optima.

\section{CONCLUDING REMARKS}

In this article, a robust formulation of structural risk optimization was presented. It was assumed that all probabilistically quantifiable uncertainties (of intrinsic or epistemic types) were incorporated in the structural reliability problem. However, results of risk optimization should be robust with respect to uncertainties of the epistemic type, which cannot be quantified probabilistically. This is in contrast to general robust optimization formulations, where no distinction needs to be made between the different types of uncertainty. Epistemic uncertainties affecting the risk optimization problem were represented as triangular fuzzy variables based on subjective judgment. The resulting fuzzy cost functions were used as objective functions in the robust optimization. In this way, the optimum solution was made robust with respect to the whole portfolio of fuzzy epistemic uncertainties.

An elementary but fundamental risk optimization problem was investigated throughout the article. Uncertainties in random variable parameters (c.o.v.), failure cost and of phenomenological nature were considered. Two levels of uncertainty in the underlying reliability problem and two costs of failure were considered. An application example involving optimization of partial safety factors for the codified design of a steel beam subject to bending was also considered.

For both the fundamental risk optimization and application problems, it was observed that the robust formulation leads to optimum structures that are more conservative and more costly than their non-robust counterparts, but also
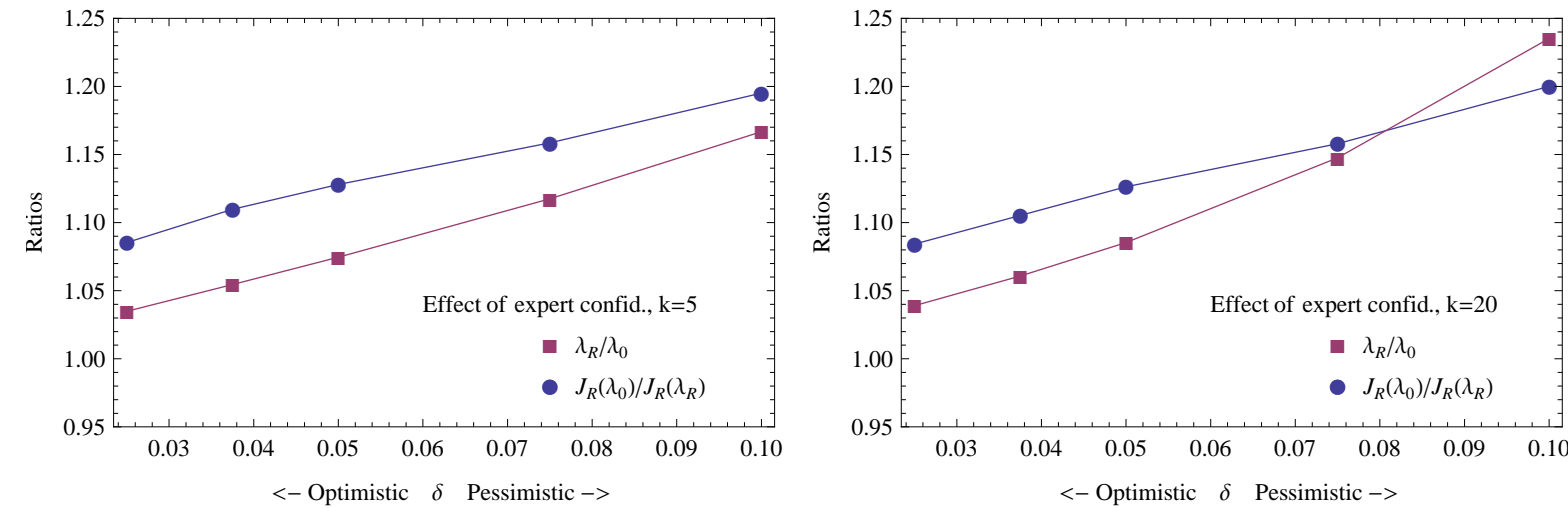

FIG. 14: Effect of expert confidence on robust optimum results.

Volume 2, Number 1, 2012 

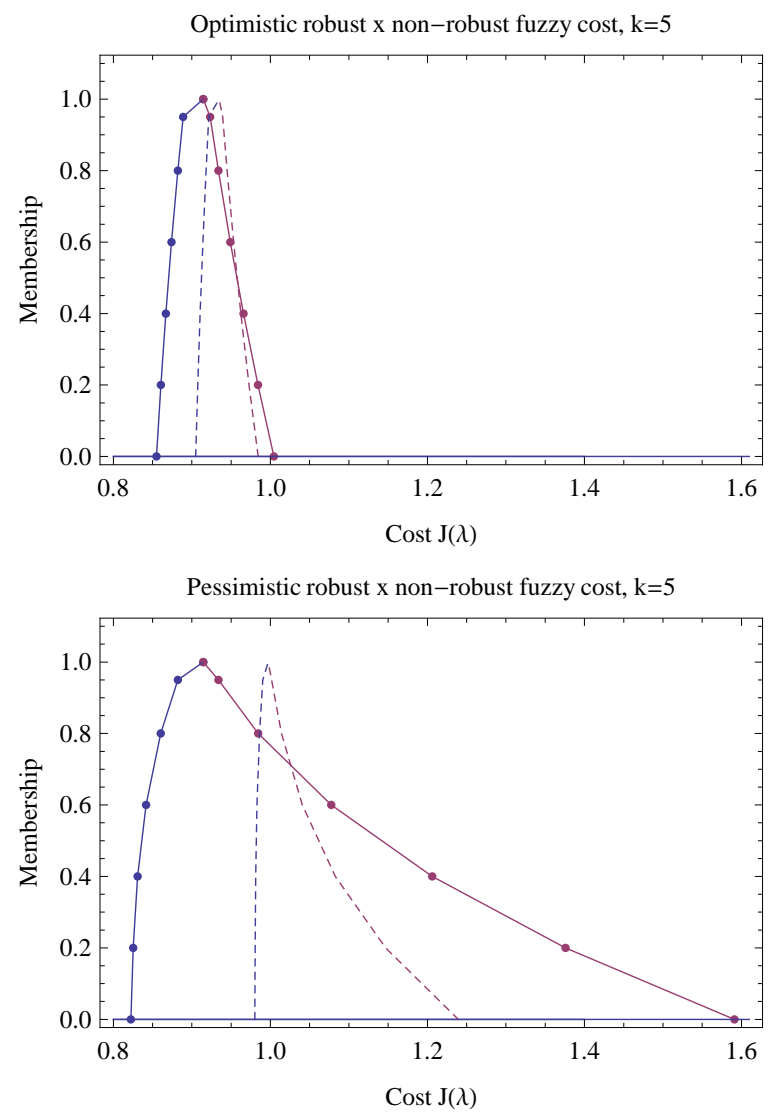

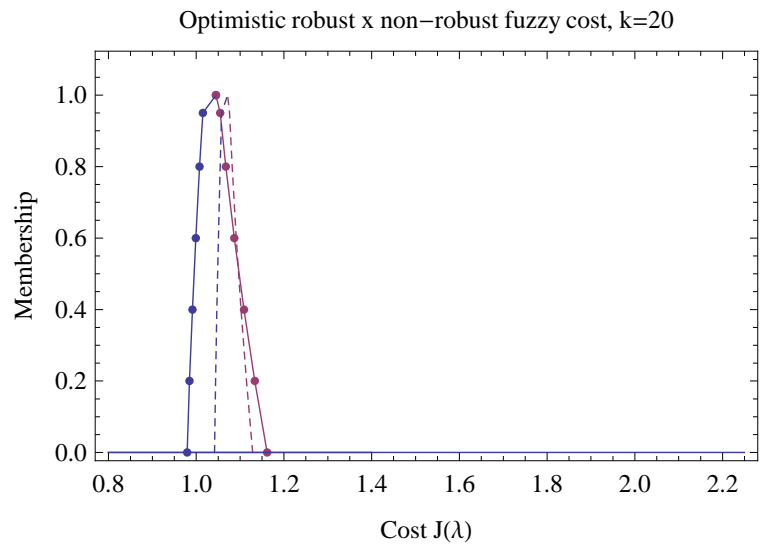

Pessimistic robust $\mathrm{x}$ non-robust fuzzy cost, $\mathrm{k}=20$

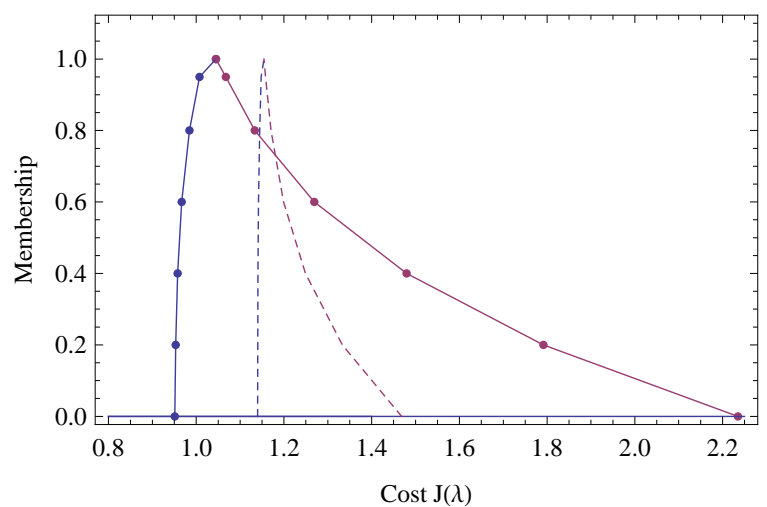

FIG. 15: Effect of expert confidence on robust and non-robust fuzzy costs.

less sensitive to epistemic uncertainties. This was observed to be increasingly so for higher levels of uncertainty in the underlying reliability problem and for higher costs of failure. It was also observed that room for improvement in robust solutions is larger when problem uncertainty is smaller. In the application example, robust optimum safety factors were found to be in reasonable agreement with the current practice, suggesting that some sort of risk optimization has been performed over the years in the gradual customization of these safety factors.

It also was shown in the article that, although risk optimization results do depend on the subjective quantification of epistemic uncertainties by individual experts, the essential result of robust optimization remains the same: the robust solution is more conservative and more expensive, but also less sensitive to epistemic uncertainties. The more pessimistic the expert, the more conservative is the robust solution he gets, in comparison to the nominal, non-robust solution.

\section{ACKNOWLEDGMENTS}

Sponsorship of this research project by the São Paulo State Foundation for Research (FAPESP) (Grant Number 2009/12099-6) and by the National Council for Research and Development (CNPq )(Grant Number 301679/20096) is greatly acknowledged.

\section{REFERENCES}

1. Beyer, H. G. and Sendhoff, B., Robust optimization-A comprehensive survey, Comput. Methods Appl. Mech. Eng., 196:3190-3218, 2007. 
2. Birge, J. R. and Louveaux, F., Introduction to Stochastic Programming, Springer, New York, 1997.

3. Kall, P. and Wallace, S. W., Stochastic Programming, Wiley, New York, 1994.

4. Schuëller, G. I. and Jensen, H. A., Computational methods in optimization considering uncertainties-An overview, Comput. Methods Appl. Mech. Eng., 198:2-13, 2008.

5. Möller, B. and Beer, M., Fuzzy Randomness-Uncertainty in Civil Engineering and Computational Mechanics, Springer, Berlin, 2004.

6. Cheng, G. D., Xu, L., and Jiang, L., Sequential approximate programming strategy for reliability-based optimization, Comput. Struct., 84(21):1353-1367, 2006.

7. Chiralaksanakul, A. and Mahadevan, S., First-Order Approximation Methods in Reliability-Based Design Optimization, ASME J. Mech. Des., 127:851-857, 2005.

8. Frangopol, D. M., Multicriteria reliability-based structural optimization, Struct. Safety, 3(1):23-28, 1985.

9. Frangopol, D. M., Structural optimization using reliability concepts, ASCE J. Struct. Eng., 111(11):2288-2301, 1985.

10. Frangopol, D. M. and Corotis, R. B., Reliability-based structural system optimization: State-of-the-art versus state-of-thepractice, Proc. of Conference on Analysis and Computation, pp. 67-78, 1996.

11. Jensen, H. A., Structural optimization of non-linear systems under stochastic excitation, Probab. Eng. Mech., 21(4):397-409, 2006.

12. Silva, M., Tortorelli, D., Norato, J., Ha, C., and Bae, H., Component and system reliability-based topology optimization using a single-loop method, Struct. Multidiscip. Optim., 41:87-106, 2010.

13. Tu, J., Choi, K. K., and Park, Y. H., A New Study on Reliability-Based Design Optimization, ASME J. Mech. Des., 121:557564, 1999.

14. Youn, B. D. and Choi, K. K., A new response surface methodology for reliability-based design optimization, Comput. Struct., 82:241-256, 2004.

15. Taflanidis, A. A. and Beck, J. L., Stochastic subset optimization for optimal reliability problems, Probab. Eng. Mech., 23(23):324-338, 2008.

16. Aktas, E., Moses, F., and Ghosn, M., Cost and safety optimization of structural design specifications, Reliab. Eng. Syst. Safety, 73:205-212, 2001.

17. Bucher, C. and Frangopol, D. M., Optimization of lifetime maintenance strategies for deteriorating structures considering probabilities of violating safety, condition, and cost thresholds, Probab. Eng. Mech., 21(1):1-8, 2006.

18. Frangopol, D. M. and Maute, K., Life-cycle reliability-based optimization of civil and aerospace structures, Comput. Struct., 81(7):397-410, 2003.

19. Haukaas, T., Unified reliability and design optimization for earthquake engineering, Probab. Eng. Mech., 23(4):471-481, 2008.

20. Streicher, H. and Rackwitz, R., Time-variant reliability-oriented structural optimization and a renewal model for life-cycle costing, Probab. Eng. Mech., 19(1-2):171-183, 2004.

21. Soltani, M. and Corotis, R. B., Failure cost design of structural systems, Struct. Safety, 5(4):239-252, 1988.

22. Beck, A. T. and Verzenhassi, C. C., Risk optimization of a steel frame communications tower subject to tornado winds, Lat. Am. J. Solids Struct., 5:187-203, 2008.

23. Beck, A. T. and Gomes, W. J. S., On structural design optimization under uncertainty and risk, IOP Conf. Ser. Mater. Sci. Eng., 10:012193, 2010.

24. Ang, A. H.-S. and Tang, W. H., Probability Concepts in Engineering: Emphasis on Applications to Civil and Environmental Engineering, 2 ed., Wiley, New York, 1999.

25. Melchers, R. E., Structural Reliability Analysis and Prediction, 2 ed., Wiley, New York, 1999.

26. Rackwitz, R., Optimization and risk acceptability based on the life quality index, Struct. Safety, 24:297-331, 2002.

27. Rackwitz, R., Life quality index revisited, Struct. Safety, 26:443-451, 2004.

28. Vanderplaats, G. N., Numerical Optimization Techniques for Engineering Design With Applications, McGraw-Hill, New York, 1984.

29. Luenberger, D. G., Linear and Nonlinear Programming, 2 ed., Kluver Academic, Norwell, MA, 2003. 
30. Vrouwenvelder, T., The JCSS probabilistic model code, Struct. Safety, 19(3):245-251, 2001.

31. Kiureghian, A. D. and Ditlevsen, O., Aleatory or epistemic: Does it matter? Struct. Safety, 31:105-112, 2009.

32. Hanss, M. and Turrin, S., A fuzzy-based approach to comprehensive modeling and analysis of systems with epistemic uncertainties, Struct. Safety, 32(6):433-441, 2010.

33. Montgomery, D. C. and Runger, G. C., Applied Statistics and Probability for Engineers, Wiley, New York, 2009.

34. Beck, A. T., de Oliveira, W. L. A., de Nardim, S., and ElDebs, A. L. H. C., Reliability-based evaluation of design code provisions for circular concrete-filled steel columns, Eng. Struct., 31:2299-2308, 2009.

35. Chaves, I. A., Beck, A. T., and Malite, M., Reliability-based Evaluation of Design Guidelines for Cold-Formed Steel-Concrete Composite Beams, J. Braz. Soc. Mech. Sci. Eng., 32:442-449, 2010.

36. American Society of Civil Engineering (ASCE), Minimum Design Loads for Buildings and Other Structures, ASCE, Reston, VA, 2006.

37. American Institute of Steel Construction (AISC), ANSI/AISC 360:2005 Specification for Structural Steel Buildings, ANSI, Chicago, 1995.

38. Ellingwood, B. and Galambos, T. V., Probability-based criteria for structural design, Struct. Safety, 1:15-26, 1982. 